\title{
Diversity Gain and MIMO Capacity for Nonisotropic Environments Using a Reverberation Chamber
}

\author{
Juan F. Valenzuela-Valdés, Antonio M. Martínez-González, and David A. Sánchez-Hernández, Senior Member, IEEE
}

\begin{abstract}
Several physical parameters of realistic nonisotropic environments have been recently emulated with reverberation chambers. In this letter, the different performance in terms of diversity gain and multiple-input-multiple-output (MIMO) capacity of the same linear MIMO array in different nonisotropic propagating scenarios is demonstrated with a reverberation chamber (RC) for the first time. This could be useful for designing antenna arrays in handset MIMO.
\end{abstract}

Index Terms-Diversity gain, multiple-input-multiple-output (MIMO) capacity, reverberation chamber (RC).

\section{INTRODUCTION}

$\mathbf{T}$ HE wireless channel has received much attention in recent years. It is well known nowadays that communication systems employing multiple-input-multiple-output (MIMO) techniques can considerably increase capacity. Channel models are typically divided into physical and nonphysical models [1], [2]. When using physical models, the channel depends on different physical parameters, such as the number of scatters (NS) or the angular spread (AS). In addition, it has also been demonstrated that system performance also depends on physical parameters [3]. In particular, system capacity has been shown to have a linear dependence to AS [4]. The influence of NS, AS, angle of arrival (AoA), or angle of departure (AoD) on system capacity has also been investigated [5]. Likewise, reverberation chambers are useful for emulating wireless propagating environments with Rayleigh-fading and isotropic scattering [6]. Recently, both polarization performance and Ricean scenarios have been properly emulated using reverberation chambers [7], [8]. Yet, it has been demonstrated by both simulations and measurements that the scattering encountered in many suburban (particularly microcellular), rural, and high speed vehicle-to-vehicle wireless communication scenarios is nonisotropic [9]-[11], resulting in a nonuniform distribution of the AoA at the receiver. In this sense, the use of absorbers has been reported to provide nonisotropic fading behavior on a scattered field chamber [12]. In [12], however, emulation of Rayleigh-fading propagation was poor and maximum power differences at diverse angles were

Manuscript received October 07, 2008; revised November 17, 2008. First published January 09, 2009; current version published April 22, 2009. This work was supported in part by the Spanish National R\&D Programme under TEC2008-05811/TEC.

J. F. Valenzuela-Valdés is with EMITE Ing, 30100 Espinardo, Spain (e-mail: juan.valenzuela@emite-ingenieria.es).

A. M. Martínez-González and D. A. Sánchez-Hernández are with the Departamento de Tecnologías de la Información y Comunicaciones, Universidad Politécnica de Cartagena, 30202 Cartagena, Spain (e-mail: david.sanchez@upct.es).

Digital Object Identifier 10.1109/LAWP.2008.2012279

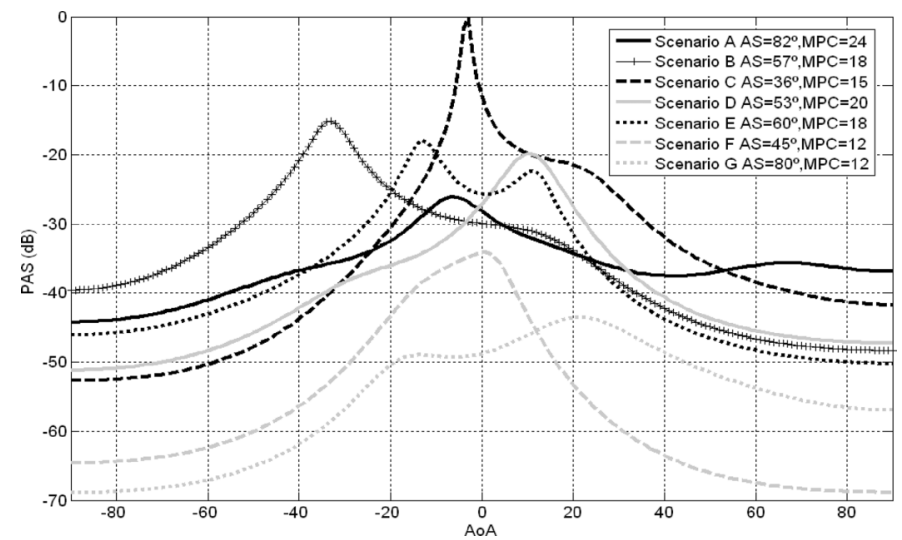

Fig. 1. Histograms of measured angle-of-arrival (AoA) using the high-resolution MUSIC algorithm.

only $5.5 \mathrm{~dB}$. In a recent letter [13], we demonstrated that with a proper setup a reverberation chamber (RC) is able to accurately emulate Rayleigh-fading scenarios with nonisotropic scattering with up to 50-dB power differences for diverse angles. In this way, several scenarios with different AS, AoA, AoD, or NS were obtained using a RC for the first time. By using these new emulated scenarios, in this letter we present their different system performance for the same MIMO linear array. While performance differences due to nonisotropic behavior were expected, they are reported here using a RC for the first time. This allows for isotropic and nonisotropic multiscenario repetitive laboratory measurements, which can certainly cut antenna development cost and research schedules. The technique is patent-protected by EMITE Ing.

\section{Measurement SetUp}

Measurements were carried out with a modified RC800 RC by Bluetest AB. Measurements were performed at $1800 \mathrm{MHz}$, and half-wave dipoles were used as MIMO array antennas. Seven different measuring scenarios (A to $G$ ) were prepared exactly as in [13]. Scenario A is an empty RC with a typical quasi-isotropic behavior. Scenarios B and C reduce the number of resolvable multipath components (MPC) by absorbing specific directions. Scenarios D and E reduce the MPC by decreasing the chamber Q-factor. Finally, scenarios F and G modify the typical reverberation chamber enclosed behavior by opening the chamber door. The technique is patent-protected by EMITE Ing. The decrease in MPC for scenarios with the open door, however, would result in an increment of the AS [13]. The nonisotropic behaviors of the diverse emulated scenarios are reproduced in Fig. 1, wherein AS is defined as in [14]. Eight different MIMO arrays were employed using spatial-diversity 
TABLE I

MEASURED DIVERSITY GAIN FOR SCENARIO F

\begin{tabular}{|c|c|c|c|c|}
\hline $\begin{array}{c}\text { MIMO } \\
\text { Array }\end{array}$ & $\begin{array}{c}\text { Spatial } \\
\text { Diversity } \\
\mathrm{D}=\mathrm{d} / \lambda\end{array}$ & $\begin{array}{c}\text { Total array } \\
\text { length } \\
\mathrm{D}_{\mathrm{I}}=\mathrm{d}_{\uparrow} / \lambda\end{array}$ & $\begin{array}{c}\text { Array } \\
\text { size } \\
(\mathrm{T} \times \mathrm{R})\end{array}$ & $\begin{array}{c}\text { IDG at } 0.1 \% \\
(\mathrm{~dB})\end{array}$ \\
\hline 1 & 0.05 & 0.10 & $3 \times 3$ & 5.01 \\
\hline 2 & 0.10 & 0.20 & $3 \times 3$ & 7.11 \\
\hline 3 & 0.15 & 0.30 & $3 \times 3$ & 9.01 \\
\hline 4 & 0.20 & 0.40 & $3 \times 3$ & 10.63 \\
\hline 5 & 0.25 & 0.50 & $3 \times 3$ & 12.24 \\
\hline 6 & 0.05 & 0.15 & $3 \times 4$ & 5.31 \\
\hline 7 & 0.10 & 0.30 & $3 \times 4$ & 9.02 \\
\hline 8 & 0.15 & 0.45 & $3 \times 4$ & 12.19 \\
\hline
\end{tabular}

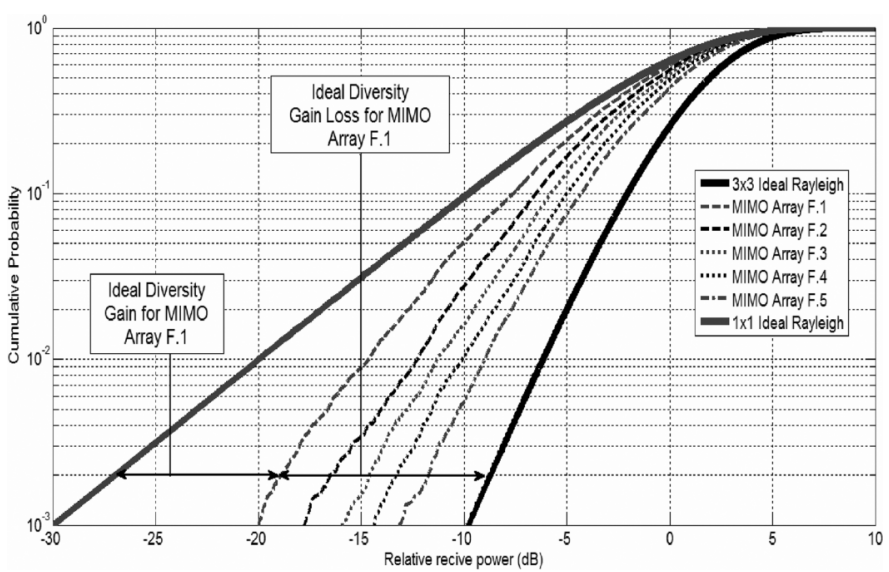

Fig. 2. Measured cumulative probability versus relative received power $(\mathrm{dB})$ for Scenario $\mathrm{F}$ and $3 \times 3 \mathrm{MIMO}$

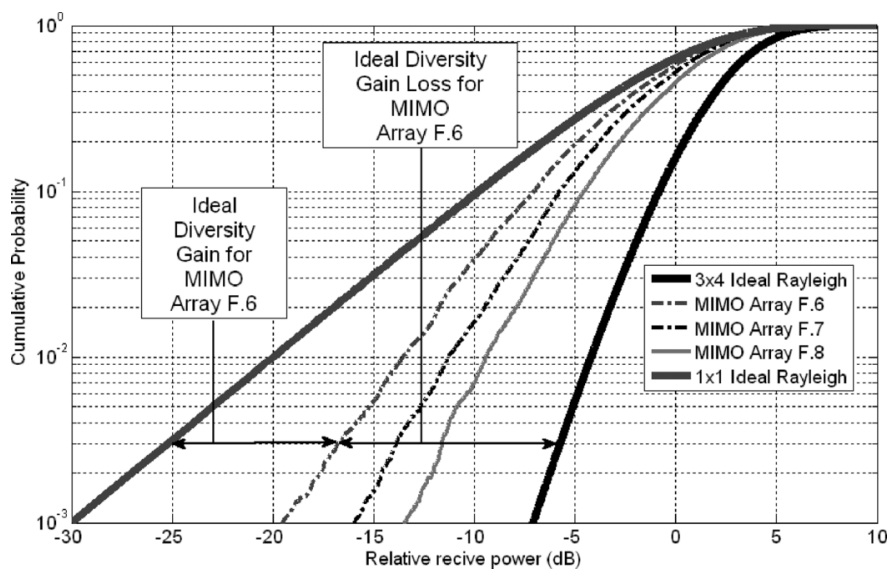

Fig. 3. Measured cumulative probability versus relative received power $(\mathrm{dB})$ for Scenario $\mathrm{F}$ and $3 \times 4 \mathrm{MIMO}$.

for each scenario. The employed $\mathrm{T} \times \mathrm{R}$ MIMO arrays are listed in Table I, where $d$ is the interelement spacing, $D=d / \lambda$, and $\mathrm{T}$ and $\mathrm{R}$ indicate the number of transmitting and receiving antennas, respectively. The measured results in this letter are obtained using the formulas described in [15]. The calibration procedures are well described in [6].

\section{DIVERSITY GAIN RESULTS}

Figs. 2 and 3 depict measured ideal diversity gain (IDG) for Scenario $\mathrm{F}$ and $3 \times 3$ and $3 \times 4 \mathrm{MIMO}$, respectively. Table I

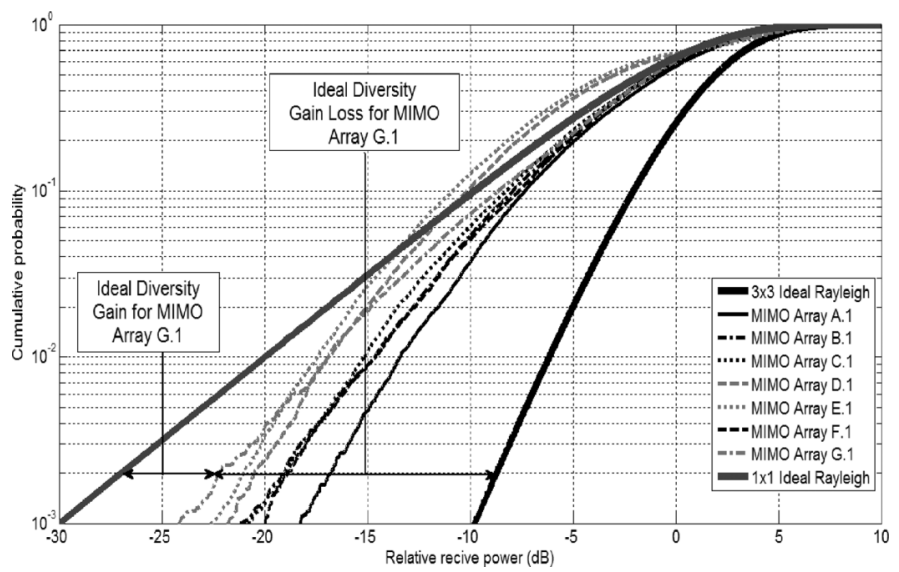

Fig. 4. Measured cumulative probability versus relative received power (dB) for all Scenarios and MIMO Array 1.

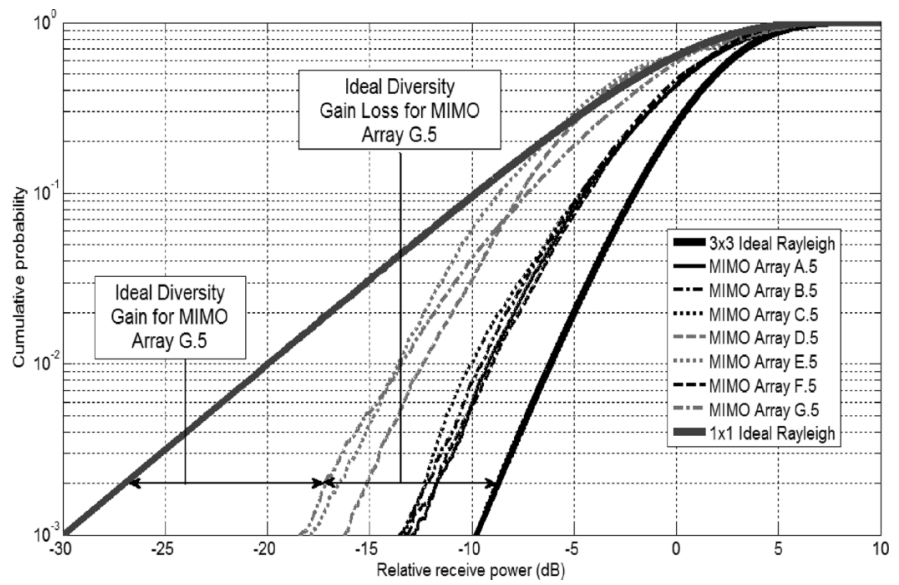

Fig. 5. Measured cumulative probability versus relative received power (dB) for all Scenarios and MIMO Array 5.

summarizes DG measured results for Scenario $\mathrm{F}$ at $0.1 \%$ probability level. Figs. 4 and 5 illustrate the measured IDG values for all scenarios with the smallest and greatest $3 \times 3$ MIMO array interelement spacing, respectively. Fig. 6 depicts measured IDG for all scenarios and MIMO Array 7, representing an intermediate $3 \times 4$ MIMO array interelement spacing. Ideal uncorrelated Rayleigh-fading results are also plotted in all figures for comparison purposes. As expected, diversity gain increases for increasing spatial separation $D$ between receive array elements. It is important to note from Table I that total array length plays an important influence on final IDG performance. As an example, IDG for the $3 \times 3$ MIMO array with $D=0.15$ and a total array length of $0.30 \lambda$ attains $9.01 \mathrm{~dB}$, which is nearly identical to the IDG obtained for the same total array length but with 4 receive array elements $(9.02 \mathrm{~dB})$. The influence of diverse isotropic behaviors on diversity gain is clearly observed from Figs. 4 and 5. While different IDG performance is clearly observed when the array size is very small (Fig. 4), for larger arrays scenarios the situation is different. From Fig. 5 (the largest MIMO array size), only the scenarios that increase AS by decreasing the cavity Q-factor perform differently. Scenarios that obtain the AS reduction with the use of absorbers (D and E) perform just like the unmodified chamber (A). The different 


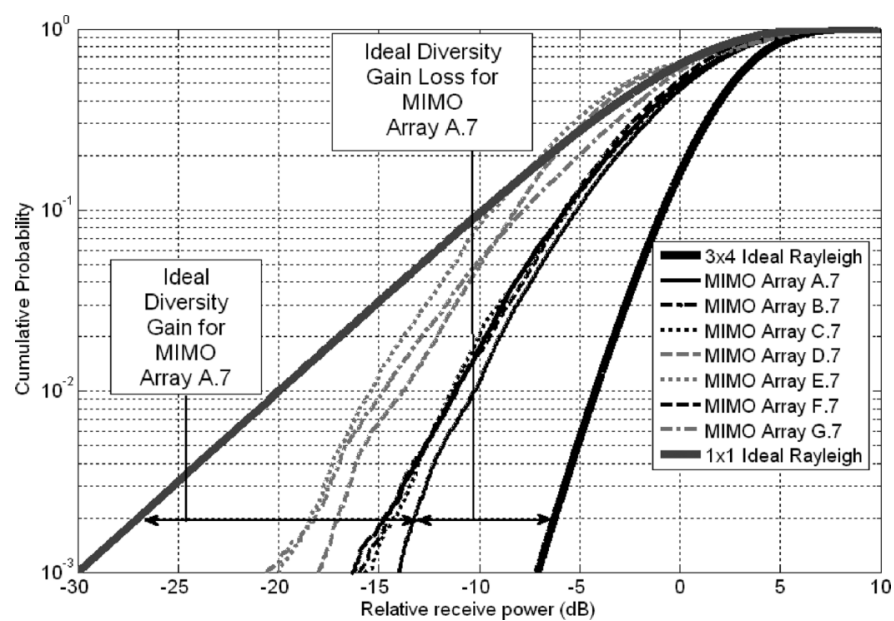

Fig. 6. Measured diversity gain versus relative received power $(\mathrm{dB})$ for all scenarios and MIMO Array 7.

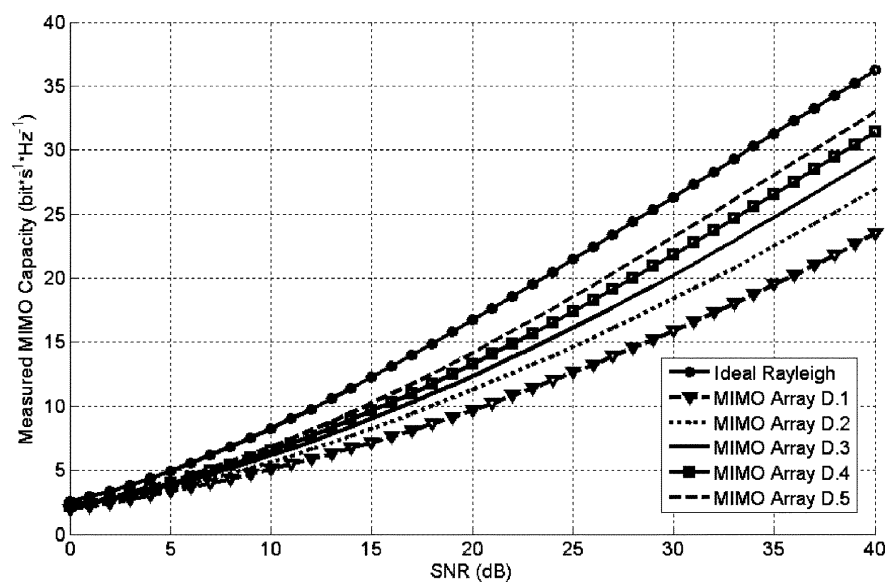

Fig. 7. Measured capacity for Scenario D and $3 \times 3$ MIMO.

behavior of scenario $\mathrm{G}$ (open door with large T-R separation) cannot be directly attributed to the large AS due to the considerably lower MPC than scenario A. This means that the use of loading for cavity Q-factor reduction is a method for emulating nonisotropic fading environments with a larger robustness than the use of absorbers. It is important to note that the differences in diversity gain performance for the diverse scenarios decrease with increasing D. Consequently, the effect of channel nonisotropicity is more important for very compact MIMO arrays, as it happens in handset MIMO. In these situations a precise emulation of the channel isotropicity would be required for accurate prediction of diversity gain performance. Finally, it is also observed from Figs. 4-6 that for specific scenarios and a high cumulative distribution function (cdf), a combination of low efficient or highly correlated antennas may behave worse than the $1 \times 1$ ideal Rayleigh case [15].

\section{MIMO CAPACITY RESULTS}

Fig. 7 shows measured capacity for scenario D and a $3 \times 3$ channel matrix. Table II summarizes measured capacity results for scenario D and $3 \times 3$ and $3 \times 4$ MIMO. Figs. 8 and 9 depict measured capacity for all scenarios and $3 \times 3$ MIMO with $D=0.05 d / \lambda$ and $D=0.25 d / \lambda$, respectively. Finally, Fig. 10
TABLE II

MEASURED MIMO CAPACITY FOR SCENARIO D

\begin{tabular}{|c|c|c|c|}
\hline $\begin{array}{c}\text { Spatial } \\
\text { Diversity } \\
\mathrm{D}=\mathrm{d} / \lambda\end{array}$ & $\begin{array}{c}\text { Total array } \\
\text { distance } \\
\mathrm{D}_{\mathrm{I}}=\mathrm{d}_{\mathrm{t}} / \lambda\end{array}$ & $\begin{array}{c}\text { Array size } \\
\text { (TxR) }\end{array}$ & $\begin{array}{c}\text { MIMO Capacity at } \\
\mathrm{SNR}=25 \mathrm{~dB} \text { for } \\
\text { scenario } \mathrm{D}(\mathrm{bit} / \mathrm{s} / \mathrm{Hz})\end{array}$ \\
\hline 0.05 & 0.10 & $3 \times 3$ & 12.91 \\
\hline 0.10 & 0.20 & $3 \times 3$ & 14.89 \\
\hline 0.15 & 0.30 & $3 \times 3$ & 16.21 \\
\hline 0.20 & 0.40 & $3 \times 3$ & 17.52 \\
\hline 0.25 & 0.50 & $3 \times 3$ & 18.61 \\
\hline 0.05 & 0.15 & $3 \times 4$ & 14.78 \\
\hline 0.10 & 0.30 & $3 \times 4$ & 17.16 \\
\hline 0.15 & 0.45 & $3 \times 4$ & 18.75 \\
\hline
\end{tabular}

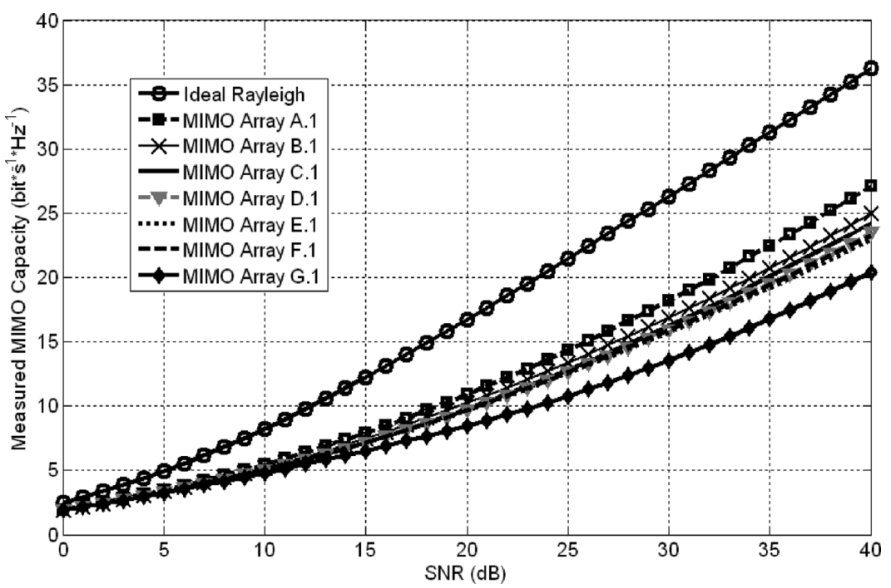

Fig. 8. Measured capacity for all Scenarios, $\mathrm{D}=0.05 \mathrm{~d} / \lambda$ and $3 \times 3 \mathrm{MIMO}$.

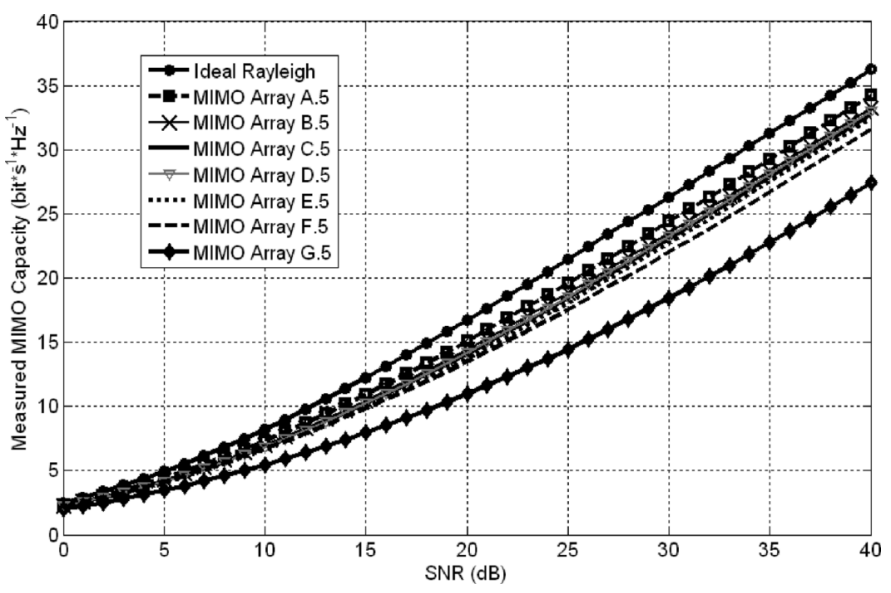

Fig. 9. Measured capacity for all Scenarios, D $=0.25 \mathrm{~d} / \lambda$ and $3 \times 3$ MIMO.

shows measured $3 \times 3$ MIMO capacities for all tested scenarios and array sizes. The ideal Rayleigh case is also depicted in Figs. 7-10 for comparison purposes, in a similar way to [16]. It is known that the interelement spacing and the number of receive elements $(\mathrm{R})$ play a dominant role on final MIMO capacity, even beyond $T / R=1$ when radiation efficiency is accounted for [15]. As expected, from Fig. 7 and Table II it is easily observed that MIMO capacity increases with increasing interelement spacing or the number of R. Interestingly, the highest attained MIMO capacity is not obtained for the largest array, but for the largest interelement spacing of the MIMO array with the largest employed $\mathrm{R}$. Thus, in contrast to what 


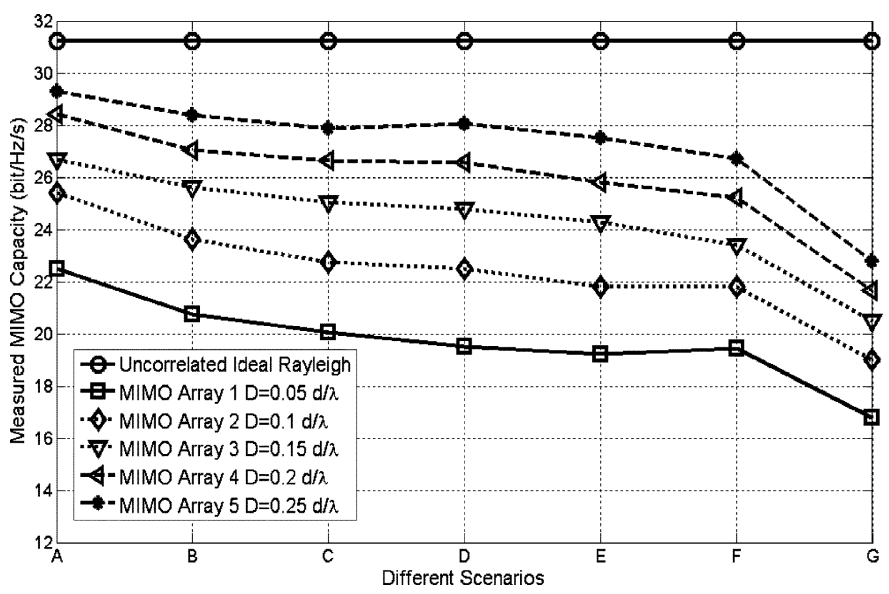

Fig. 10. Measured capacity for all scenarios and $3 \times 3$ MIMO.

happened to IDG, the number of receive elements plays a more important role on MIMO capacity performance than the interelement spatial separation. The dominant role of interelement spacing and number of receive elements on MIMO capacity is also clearly observed in Figs. 8 and 9.

Similarly, the AS has been demonstrated to have a significant effect on system capacity [17]. In addition, it has also been demonstrated that an AS of $60^{\circ}$ is sufficient to achieve a system capacity close to the limit that corresponds to isotropic scattering [18]. The AS of a uniform distribution of the power in all angles corresponds to $\approx 104^{\circ}$ [9]. The AS is in fact confirmed to have an important effect on final MIMO capacity from Fig. 10. The reduction of MIMO capacity in scenarios F and G (open door) despite their increased AS is due to their considerably reduced power angular spectrum with respect to the other scenarios. Interestingly, it is also clearly observed from this figure that the effect of AS on MIMO capacity is not affected by the array size, unlike what happened to IDG. This once again confirms the existing tradeoff between diversity and multiplexing gain.

\section{CONCLUSION}

Measured diversity gain and MIMO capacities for diverse nonisotropic Rayleigh-fading environments have been reported using a modified reverberation chamber for the first time. Diversity gain with nonisotropic environments has been demonstrated to strongly depend upon the interelement spacing. In contrast, MIMO capacity has been demonstrated to depend significantly on number of receive elements, but the effect of AS on final capacity has been found to be independent of interelement spacing. In addition, it has also been demonstrated that the use of loading for cavity Q-factor reduction is a method for emulating nonisotropic fading environments with a larger robustness than the use of absorbers. Since the channel statistics depend on the mobile direction of travel in nonisotropic environments, unlike for isotropic scattering environments, future research is envisaged to emulate the direction $\phi_{v}$, velocity $|\mathrm{v}|$, and shaped Doppler power spectral density of the mobile user using reverberation chambers. The developed techniques for nonisotropic emulation of MIMO performance using modified reverberation chambers are patent-protected by EMITE Ing. The modified chamber operates in both reverberating and nonreverberating modes.

\section{REFERENCES}

[1] K. You, Q. Li, and M. Ho, "Measurement investigation of tap and cluster angular spread at $5.2 \mathrm{GHz}, "$ IEEE Trans. Antennas Propag., vol. 53, no. 7, pp. 2156-2160, Jul. 2005.

[2] C. Orlenius, M. Franzén, P. S. Kildal, and U. Carlberg, "Investigation of heavily loaded reverberation chamber for testing wideband wireless units," in Proc. IEEE Antennas Propag. Soc. Int. Symp., Jul. 2006, pp. 3569-3572.

[3] O. Spencer, M. Rice, B. Jeffs, and M. Jensen, "Indoor wideband time/angle of arrival multipath propagation results," in Proc. IEEE Veh. Technol. Conf., May 1997, vol. 3, pp. 1410-1414.

[4] A. S. Y. Poon, D. N. C. Tse, and R. W. Brodersen, "Impact of scattering on the capacity, diversity, and propagation range of multiple-antenna channels," IEEE Trans. Inf. Theory, vol. 52, no. 3, pp. 1087-1100, Mar. 2006.

[5] N. Czink, M. Herdin, H. Ozcelik, and E. Bonek, "Number of multipath clusters in indoor MIMO propagation," Electron. Lett., vol. 40, no. 23, pp. 1498-1499, Nov. 2004

[6] K. Rosengren and P.-S. Kildal, "Radiation efficiency, correlation, diversity gain, and capacity of a six monopole antenna array for a MIMO system: Theory, simulation and measurement in reverberation chamber," in Proc. IEE, Microw. Antennas Propag., Feb. 2005, vol. 152 , no. 1 , pp. 7-16.

[7] P. S. Kildal and C. Carlsson, "Detection of a polarization imbalance in reverberation chambers and how to remove it by polarization stirring when measuring antenna efficiencies," Microw. Opt. Technol. Lett., vol. 34, no. 2, pp. 145-149, Jul. 2002.

[8] C. L. Holloway, D. A. Hill, J. M. Ladbury, P. F. Wilson, G. Koepke, and J. Coder, "On the use of reverberation chambers to simulate a Rician radio environment for the testing of wireless devices," IEEE Trans. Antennas Propag., vol. 54, no. 11, pp. 3167-3177, Nov. 2006.

[9] R. Iqbal and T. D. Abhayapala, "On statistics of the mobile Rayleigh fading channel in non-isotropic scattering environments," in Proc. IEEE Int. Symp. Commun. Inf. Tech., 2007, pp. 814-818.

[10] K. Anim-Appiah, "Complex envelope correlations for non-isotropic scattering," Electron. Lett., vol. 34, no. 9, pp. 918-919, Apr. 1998.

[11] J. Dulmage and M. P. Fitz, "Nonisotropic fading channel model for the highway environment," IEEE Veh. Technol. Mag., vol. 2, no. 4, pp. 12-18, Dec. 2007.

[12] M. Otterskog and K. Madsén, "On creating a nonisotropic propagation environment inside a scattered field chamber," Microw. Opt. Technol. Lett., vol. 43, no. 3, pp. 192-195, Nov. 2005.

[13] J. F. Valenzuela-Valdés, A. M. Martínez-González, and D. A. SánchezHernández, "Emulation of MIMO nonisotropic fading environments using reverberation chambers," IEEE Antennas Wireless Propag. Lett., vol. 7, pp. 325-328, 2008.

[14] B. H. Fleury, "First- and second-order characterization of direction dispersion and space selectivity in the radio channel," IEEE Trans. Inf. Theory, vol. 46, no. 6, pp. 2027-2044, Sep. 2000.

[15] J. F. Valenzuela-Valdés, M. A. García-Fernández, A. M. MartínezGonzález, and D. A. Sánchez-Hernández, "The influence of efficiency on receive diversity and MIMO capacity for Rayleigh-fading channels," IEEE Trans. Antennas Propag., vol. 56, no. 5, pp. 1444-1450, May 2008.

[16] A. F. Molisch, M. Steinbauer, M. Toeltsch, E. Bonek, and R. S. R. S. Thoma, "Capacity of MIMO systems based on measured wireless channels," IEEE J. Sel. Areas Commun., vol. 20, pp. 561-569, Apr. 2002.

[17] M. A. Saeed, B. M. Ali, M. Ismail, and S. Khatun, "Effects of spatial correlation and associated parameters on the capacity of MIMO fading channels," in Proc. IEEE Int. Conf. Networks, Nov. 2005, vol. 2, p. 6.

[18] B. S. Khatri, "Effect on capacity of clustering in indoor MIMO channels," in Proc. IEE Seminar on MIMO: Commun. Syst. from Concept to Implementations, Dec. 2001, pp. 15/1-15/7. 